\title{
DESIGN AND DEVELOPMENT OF ARM-BASED CONTROL SYSTEM FOR NURSING BED
}

\author{
WANG Shujie and CHENG Wushan \\ College of Mechanical Engineering, Shanghai University of Engineering Science, \\ Shanghai, China
}

\begin{abstract}
This paper introduces a kind of ARM embedded system as the control systemof the nursing bed.The embedded control system takes the ARM9 S3C2440 chip as the core of data processing. The design ofthe control system includes hardware design, software design and PC monitoring system design.
\end{abstract}

\section{KEYWORDS}

Nursing Bed; ARM9; Embedded Control System.

\section{INTRODUCTION}

With the increasing number of the elderly population in China, the aging phenomenon is becoming increasingly serious. Nursing, health care and rehabilitation of the elderly has become one of the important issues of social concern. How to solve the problem of nursing care for the elderly who cannot take care of themselves because of physical weakness or completely bedridden, is a common problem faced by the countries with aging population[1]. The electric nursing bed has the advantages of convenient operation, time saving and labor saving, so it is increasingly becoming the research and development focus of the world as well as various research institutes and businesses [2].

This paper presents a kind of ARM embedded system as the control system of the nursing bed. The embedded control system takes the ARM9 S3C2440 chip as the core of data processing and it can communicate with PC through the serial port. In this paper, the hardware design, software design and PC monitoring system design are presented based on the related functions of the nursing bed.

\section{Control System of The NURSING BED}

The nursing bed mentioned in this paper integrates multiple functions, including rehabilitation and nursing function. The control system as the core of the nursing bed, its control ability directly affects the realization of rehabilitation and nursing function of the nursing bed, so we should to ensure the reliability and stability of the control system [3]. This design applies ARM embedded system in the control system of the nursing bed. Each function of the nursing bed is realized by the motor driving the corresponding bed plate. We can achieve the corresponding functions and the reset of corresponding function by controlling the rotation of the motor. It is not only meets the needs of the elderly, but also reduces the workload of medical staff. The control system of the 
nursing bed mainly includes hardware design, software design and PC monitoring system design. The overall framework of the control system of the nursing bed is shown in Figure 1:

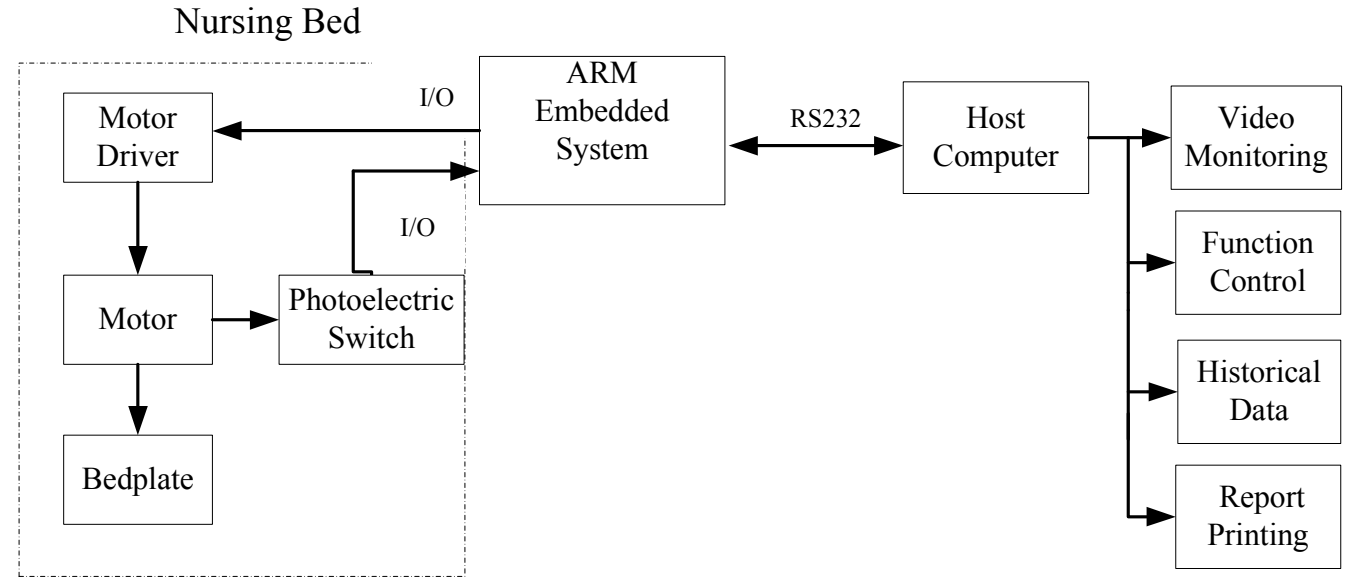

Fig. 1The overall framework of the control system

In this system, the stability of the control system is achieved by using modular design. We develop a high speed CPU based on ARM kernel as the main control unit of the system. Through the embedded system, the multifunction and multitask of the nursing bed is completed. The design can solve the communication of the control system based on ARM, complete the seamless connection between the control system and the external equipment, and ensure the good scalability of the system.

\subsection{Hardware Design of the Nursing Bed Control System}

The hardware structure of the control system of the nursing bed takes the S3C2440 chip as the core, and realizes the control of the nursing bed. The hardware structure mainly includes the core processing module and power supply module. It mainly accomplishes the signal acquisition, data acquisition of motor and communication with the host computer. The hardware framework of the control system is shown in Figure 2. When the user needs rehabilitation or nursing, the corresponding functions needed are converted into switch signals. Then the switch signals are transmitted to the main control chip S3C2440A by I/O ports. After the switch signals are processed by the core chip, the corresponding function are accomplished by the motor driving the bedplates, meanwhile, the related parameters of each function are uploaded to the host computer through the RS232communication.

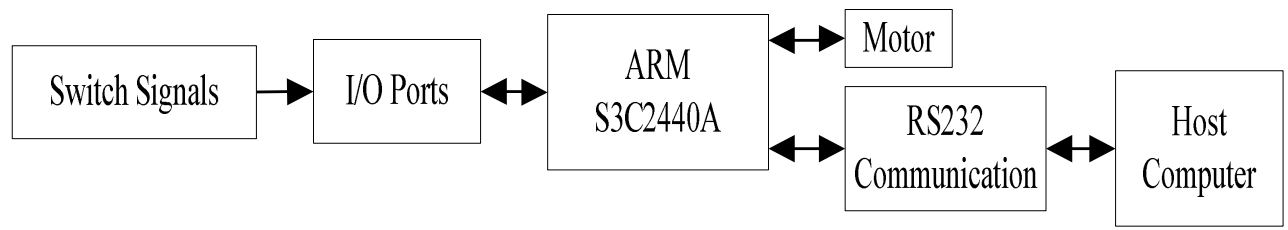

Fig. 2 The hardware framework of the control system 
In the hardware design, we designed the development board based on S3C2440A from the consideration of various aspects of the system. ARM development board consists of two parts: core board and backplane. The core board and backplane are connected by a socket connector. The advantage of this design is that if you need to change the circuit or to expand the function of the system, you only need to redesign the backplane, which can reduce the cost. The design of core board circuit includes a power supply circuit board, SDRAM, NAND FLASH, NOR FLASH and other parts of the circuit [4]. The design of backplane circuit includes the power supply circuit, the minimum system circuit, RS232 communication circuit and USB circuit. After the circuit diagrams of core board and backplane are completed, we carry on the reasonable layout of the components and wiring, and then complete the design of the PCB boards. The designed PCB boards have strong anti-jamming characteristics and good electromagnetic compatibility.

\subsection{Software Design of the Nursing Bed Control System}

The system software is very important for system function, because it decides whether system function can be implemented correctly. The quality of software determines the realization of function and the stability of the system. In order to facilitate the development of software and the extension of the function, the design idea of modularization is adopted in the software development and design. Therefore the function of the system can be adjusted in real time, so that the system has better real-time and stability.

After writing embedded control program on ARM development board, the control of the moving parts and the transmission of the relevant parameters of motor can be realized. The main modules of the program includeRS232 communication program, system function program and PC monitoring program.

The RS232 communication program is designed to realize the communication between the master control unit and the host computer, and to accomplish the signal reception of the host computer and the upload of the data collected by the slave computer in time [5].The serial communication between host computer and slave computer can be realized in two different ways, interrupt and query [6].In this system, the way of interrupt is used to realize the communication between the two. The flow chart of serial data sending and receiving is shown in figure 3. After starting system program, the RS232 communication port initialization settings are completed first, and then enter the interrupt wait state. When PC sends control signals to the main control unit, the slave computer responses to the interrupt signal, and then jumps into the interrupt program to execute the corresponding function. After the implementation of the program is completed, it will come back to the main program, and continue to maintain the interrupt wait state. When the master control unit sends data to the host computer, it will request to interrupt from the host computer. After the host computer responding to the interrupt, it will send a flag to the master control unit for sending the data to the beginning. After the master control unit receives the transmission flag, then begins to send data [7]. 

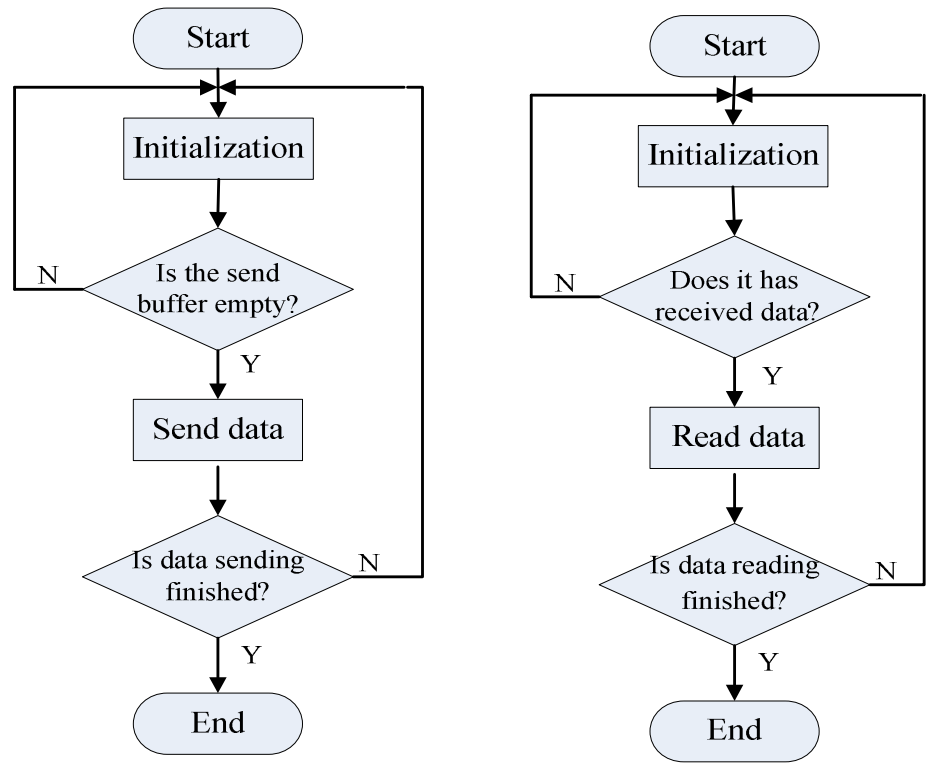

Fig. 3 The flow chart of serial data sending and receiving

Program design of system mainly includes the program design of timer module, motor function module, external interrupt module and serial port module. In this system, the control mode of the motor is controlled by position, which drives the motor to rotate by providing the direction signal and the pulse signal for the motor. Therefore, in this system, the timer module program is designed on the one hand to provide the system clock, on the other hand, to provide the motor pulse frequency. The program design of motor function module is to achieve the rotation of the motor through setting the motor direction signal and pulse signal. The software framework of the control system is shown in Figure 4.

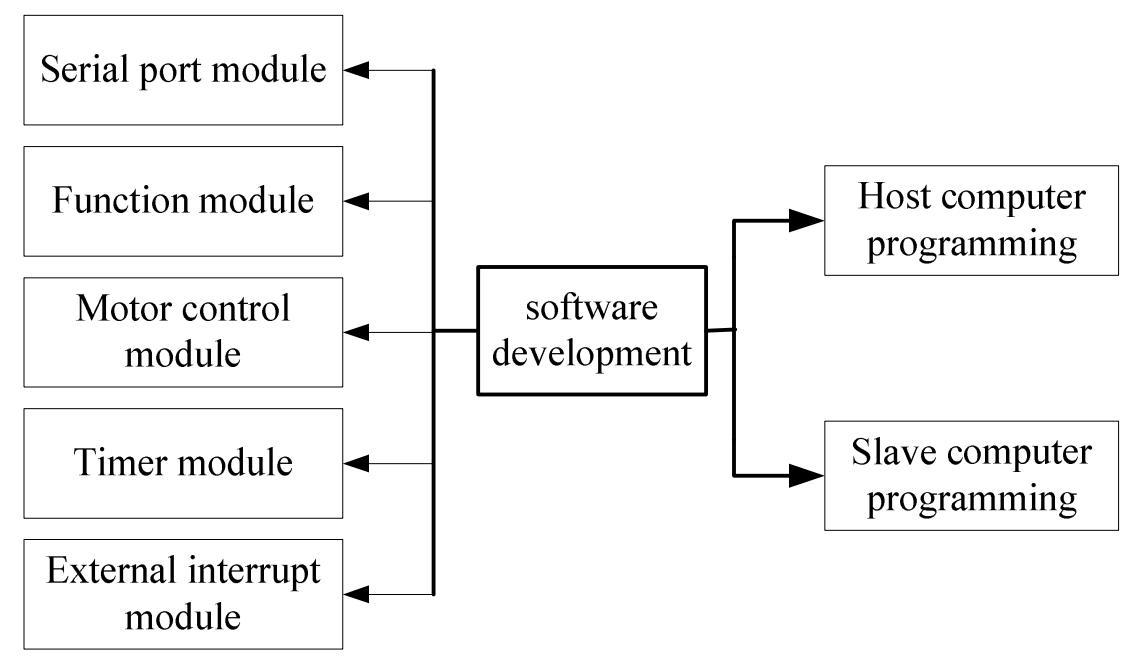

Fig. 4 The software framework of the control system 


\subsection{PC Monitoring System of the Nursing Bed}

The PC monitoring system of the nursing bed is a virtual reality operating system based on C\# programming language. It includes video monitoring, nursing and rehabilitation operation function, historical data display, report forms printing, and vital sign parameter measurement and display. It can realize the function of centralized operation and decentralized control for the nursing bed, and can respond to the dynamic needs of patients in real time. The virtual reality operating system can be used to monitor the status of a single medical staff in the general monitoring room monitoring the multiple units of the nursing beds. Therefore it can reduce the workload of medical staff and improve work efficiency. We use virtual reality technology to achieve animation operation, video response, dynamic monitoring of the state of the patient's bed, and we can control the nursing bed in real time to meet the needs of patients. The interface of PC monitoring system is shown in Figure 5.

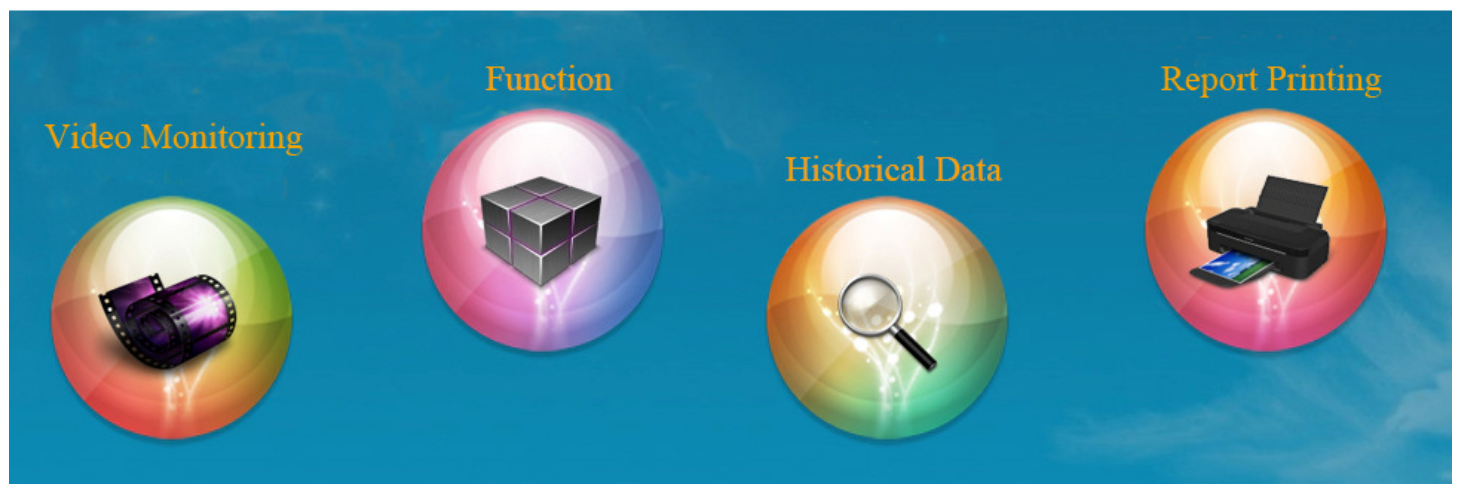

Fig. 5 The interface of PC monitoring system

\section{Conclusions}

The control system of the nursing bed presented in this paper can achieve the requirements of intelligent, low power consumption and scalable hardware and software. Software design uses modular design, which is conducive to the further upgrading and improvement of the function of nursing bed, and can improve the design efficiency of the system. The PC monitoring system can monitor the status of the nursing bed in real time. It has high reliability, good expansibility, and it can meet the needs of different patients, which is of great significance to the long-term development of the industry.

\section{ACKNOWLEDGEMENTS}

This work is supported by the Shanghai Science and Technology Committee.

\section{REFERENCES}

[1] Jiang H, Wang W and Liu Y P. Research and development of multifunctional nursing bed [J]. Development and application of SCM, 2006,22(7-2):117-120.

[2] Zhang B H. The design and research of multifunctional rehabilitation bed [D]. TianjinUniversity of Science and Technology, 2009. 
International Journal of Computer Science \& Engineering Survey (IJCSES) Vol.7, No.2, April 2016

[3] Guo J L. Design and research of embedded control system for lower limb rehabilitation robot [D]. Hebei University of Technology, 2013.

[4] Li S L, Feng J, and Liu W J. The hardware design of embedded system based on S3C2440A [J]. Journal of Huanggang Normal University,2009, 29(3):37-41.

[5] Wang Y. Development of multifunctional electric nursing bed control system [J]. Manufacturing automation, 2009,31(6):70-72.

[6] He Q Y. ARM based remote monitoring control system of the nursing service robot [D]. Nanchang University, 2011.

[7] SAMSUNG ELECTRONICS Datasheet:S3C2440A USER'MANUAL Revision 0.12,2004.

\section{Authors}

Wang Shujie was born in 1992, the postgraduate of Shanghai University of Engineering Science. Her present research interest is embedded control system.

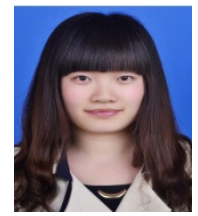

